\title{
Vertical Contracting with Informational Opportunism
}

\author{
By VIANNEY DEQUIEDT AND DAVID MARTIMORT
}

ONLINE APPENDIX

\section{ApPendix B: The Revelation Principle under Bilateral Contracting}

Section B.B1 describes the most general class of bilateral contracts that could be envisioned in our framework. Section B.B2 derives a set of new incentive constraints that apply to the principal if one wants to characterize implementable allocations. Because this Appendix is of general interest beyond the specific vertical contracting problem under scrutiny, we will slightly generalize the presentation and allow for $n \geq 2$ agents. For ease of notations, we denote the principal's and the agents' utility functions respectively as:

$$
\mathcal{V}(\mathbf{q}, \mathbf{t})=\sum_{i=1}^{n} t_{i}-C(\mathbf{q}) \text { and } \mathcal{U}_{i}\left(\mathbf{q}, \mathbf{t}, \theta_{i}\right)=u_{i}\left(\mathbf{q}, \theta_{i}\right)-t_{i}
$$

\section{B1. Mechanisms and Timing}

In the main text, our presentation of the bilateral contracting setup focused on deterministic nonlinear prices because such mechanisms echo real world practices in vertical contracting arrangements and because they have been extensively used in the vertical contracting literature. In this Appendix, we extend the scope of our analysis by allowing for stochastic mechanisms and more general communication protocols.

A bilateral mechanism $\mathcal{B}_{i}$ ruling the relationship between the principal and agent $A_{i}$ is a triplet consisting of a message space $\mathcal{M}_{i}^{a}$ for $A_{i}$ (with generic message $m_{i}^{a}$ ), a message space $\mathcal{M}_{i}^{p}$ for the principal (with generic message $\left.m_{i}^{p}\right)$ and a (joint) distribution $\sigma_{i}\left(m_{i}^{a}, m_{i}^{p}\right)$ of agent $A_{i}$ 's payment and output on the compact set $\mathcal{Q}_{i} \times \mathcal{T}_{i}$. We will denote $d \sigma_{i}\left(q_{i}, t_{i} \mid m_{i}^{a}, m_{i}^{p}\right)$ the corresponding measure. For future reference, let $\Delta(\mathcal{E})$ denote the set of probability measures on any arbitrary set $\mathcal{E}$.

Let $\mathbf{m}^{a}=\left(m_{1}^{a}, \ldots, m_{n}^{a}\right) \in \mathcal{M}^{a}=\mathcal{M}_{1}^{a} \times \ldots \times \mathcal{M}_{n}^{a}$ be an array of messages sent by the agents to the principal and $\mathbf{m}^{p}=\left(m_{1}^{p}, \ldots, m_{n}^{p}\right) \in \mathcal{M}^{p}=\mathcal{M}_{1}^{p} \times \ldots \times \mathcal{M}_{n}^{p}$ be an array of messages sent by the principal to each of his agents respectively. Let also $\mathcal{B}=\left(\mathcal{B}_{1}, \ldots, \mathcal{B}_{n}\right)$ be an array of bilateral mechanisms with the corresponding array of distributions $\left(\sigma_{1}\left(m_{1}^{a}, m_{1}^{p}\right), \ldots, \sigma_{n}\left(m_{n}^{a}, m_{n}^{p}\right)\right)$ induced by the respective messages of the agents and the principal in each bilateral relationship.

Payoffs are defined as expectations over the relevant mixtures. For instance, we denote agent $A_{i}$ 's expected payoff when his type is $\theta_{i}$, the messages are $\mathbf{m}^{a}=\left(m_{1}^{a}, \ldots, m_{n}^{a}\right)$ and $\mathbf{m}^{p}=\left(m_{1}^{p}, \ldots, m_{n}^{p}\right)$, and the distribution of payments and outputs is induced by the bilateral contracts $\left(\sigma_{1}\left(m_{1}^{a}, m_{1}^{p}\right), \ldots, \sigma_{n}\left(m_{n}^{a}, m_{n}^{p}\right)\right)$ by:

$$
\mathcal{U}_{i}\left(\sigma_{1}\left(m_{1}^{a}, m_{1}^{p}\right), \ldots, \sigma_{n}\left(m_{n}^{a}, m_{n}^{p}\right), \theta_{i}\right)=\int \mathcal{U}_{i}\left(\mathbf{q}, \mathbf{t}, \theta_{i}\right) d \sigma_{1}\left(t_{1}, q_{1} \mid m_{1}^{a}, m_{1}^{p}\right) \ldots d \sigma_{n}\left(t_{n}, q_{n} \mid m_{n}^{a}, m_{n}^{p}\right) .
$$

We will sometimes use the notation $\mathcal{U}_{i}\left(\sigma_{i}\left(m_{i}^{a}, m_{i}^{p}\right), \sigma_{-i}\left(m_{-i}^{a}, m_{-i}^{p}\right), \theta_{i}\right)$ to isolate the role played by the bilateral mechanism $\mathcal{B}_{i}$. Similarly, the principal's expected payoff writes as $\mathcal{V}\left(\sigma_{1}\left(m_{1}^{a}, m_{1}^{p}\right), \ldots, \sigma_{n}\left(m_{n}^{a}, m_{n}^{p}\right)\right)$ where:

$$
\mathcal{V}\left(\sigma_{1}\left(m_{1}^{a}, m_{1}^{p}\right), \ldots, \sigma_{n}\left(m_{n}^{a}, m_{n}^{p}\right)\right)=\int \mathcal{V}(\mathbf{q}, \mathbf{t}) d \sigma_{1}\left(t_{1}, q_{1} \mid m_{1}^{a}, m_{1}^{p}\right) \ldots d \sigma_{n}\left(t_{n}, q_{n} \mid m_{n}^{a}, m_{n}^{p}\right)
$$

The contracting game generalizes that presented in the main text. First, agents privately learn their types. Second, the principal (publicly) offers the bilateral contracts $\mathcal{B}$. Third, each agent $A_{i}$ accepts or refuses his own offer $\mathcal{B}_{i}$. If he refuses, he gets a payoff that is normalized to zero. Fourth, agents simultaneously send their messages $\mathbf{m}^{a}=\left(m_{1}^{a}, \ldots, m_{n}^{a}\right)$ to the principal. Finally, knowing the vector of agents' messages $\mathbf{m}^{a}$, the principal optimally chooses to send back the messages $\mathbf{m}^{p *}\left(\mathbf{m}^{a}\right)=\left(m_{1}^{p *}\left(\mathbf{m}^{a}\right), \ldots, m_{n}^{p *}\left(\mathbf{m}^{a}\right)\right)$ in each relationship. 


\section{B2. The Principal's Ex Post Incentive Constraints}

We now characterize the set of allocations that can be achieved as perfect Bayesian equilibria of the contracting game when the principal offers any possible menu of bilateral contracts $\mathcal{B}$.

Let $m_{i}^{a *}\left(\theta_{i}\right)$ be agent $A_{i}$ 's optimal reporting strategy (which is possibly mixed) when his type is $\theta_{i}$. Thus, $m_{i}^{a *}$ maps $\Theta$ into $\Delta\left(\mathcal{M}_{i}^{a}\right)$. Let also $\mathbf{m}^{a *}(\theta)=\left(m_{1}^{a *}\left(\theta_{1}\right), . ., m_{n}^{a *}\left(\theta_{n}\right)\right)$ be the array of such strategies. We denote by supp $m_{i}^{a *}\left(\theta_{i}\right)$ the support of $m_{i}^{a *}\left(\theta_{i}\right)$, i.e., the set of messages sent with positive probability by type $\theta_{i}$. Let $\mathbf{m}^{p *}\left(\mathbf{m}^{a}\right)$ be principal $P^{\prime}$ s optimal pure reporting strategy when he observes the messages $\mathbf{m}^{a}$. Thus, $\mathbf{m}^{p *}$ maps $\mathcal{M}^{a}$ into $\mathcal{M}^{p}$. Observe that the principal does not randomize among the messages he sends back to the agents at this last stage of the game. This restricts the possible continuation equilibria (we make this restriction explicit in the definition of Lemma B.1 below) but is consistent with the idea that the relationship is run with bilateral contracts and the principal cannot indirectly correlate plays in those relationships by himself correlating the messages he sends back to the agents.

We denote agent $A_{i}$ 's expected payoff when the vector of types is $\left(\theta_{i}, \theta_{-i}\right)$, other agents play the (possibly) mixed strategies $m_{-i}^{a *}\left(\theta_{-i}\right)$, the principal plays $\mathbf{m}^{p *}\left(\mathbf{m}^{a}\right)$ and agent $A_{i}$ sends message $m_{i}^{a}$ by:

$$
\begin{aligned}
& \left.\mathcal{U}_{i}\left(\sigma_{i}\left(m_{i}^{a}, m_{i}^{p *}\left(m_{i}^{a}, m_{-i}^{a *}\left(\theta_{-i}\right)\right)\right), \sigma_{-i}\left(m_{-i}^{a *}\left(\theta_{-i}\right), m_{-i}^{p *}\left(m_{i}^{a}, m_{-i}^{a *}\left(\theta_{-i}\right)\right)\right)\right), \theta_{i}\right) \\
& =\int \mathcal{U}_{i}\left(\sigma_{1}\left(m_{1}^{a}, m_{1}^{p}\left(m_{i}^{a}, m_{-i}^{a *}\left(\theta_{-i}\right)\right)\right), \ldots, \sigma_{n}\left(m_{n}^{a}, m_{n}^{p}\left(m_{i}^{a}, m_{-i}^{a *}\left(\theta_{-i}\right)\right)\right), \theta_{i}\right) \\
& \times d m_{1}^{a *}\left(m_{1}^{a} \mid \theta_{1}\right) \ldots d m_{i-1}^{a *}\left(m_{i-1}^{a} \mid \theta_{i-1}\right) d m_{i+1}^{a *}\left(m_{i+1}^{a} \mid \theta_{i+1}\right) \ldots d m_{n}^{a *}\left(m_{n}^{a} \mid \theta_{n}\right) .
\end{aligned}
$$

Finally, we denote the principal's expected payoff when the vector of types is $\theta$, agents play the (possibly) mixed strategies $\mathbf{m}^{a *}(\theta)$ and the principal plays $\mathbf{m}^{p *}\left(\mathbf{m}^{a}\right)$ by:

$$
\begin{gathered}
\mathcal{V}\left(\sigma_{1}\left(m_{1}^{a *}\left(\theta_{1}\right), m_{1}^{p *}\left(\mathbf{m}^{a *}(\theta)\right)\right), \ldots, \sigma_{n}\left(m_{n}^{a *}\left(\theta_{n}\right), m_{n}^{p *}\left(\mathbf{m}^{a *}(\theta)\right)\right)\right) \\
=\int \mathcal{V}\left(\sigma_{1}\left(m_{1}^{a}, m_{1}^{p *}\left(\mathbf{m}^{a}\right)\right), ., \sigma_{n}\left(m_{n}^{a}, m_{n}^{p *}\left(\mathbf{m}^{a}\right)\right) d m_{1}^{a *}\left(m_{1}^{a} \mid \theta_{1}\right) \ldots d m_{n}^{a *}\left(m_{n}^{a} \mid \theta_{n}\right) .\right.
\end{gathered}
$$

For a given set of bilateral contracts $\mathcal{B}=\left(\mathcal{B}_{1}, \ldots, \mathcal{B}_{n}\right)$, a continuation equilibrium where offers are accepted (sometimes in short a continuation equilibrium) is described as follows.

LEMMA B.1: Fix any arbitrary set of bilateral mechanisms $\mathcal{B}$. A continuation equilibrium is a pair $\left(\mathbf{m}^{a *}, \mathbf{m}^{p *}\right)$ such that:

- The agents' reporting strategies $\mathbf{m}^{a *}=\left(m_{1}^{a *}, . ., m_{n}^{a *}\right)$ form a Bayesian equilibrium given the principal's optimal choice $\mathbf{m}^{p *}$ if and only if for any $m_{i}^{a} \in \operatorname{supp} m_{i}^{a *}\left(\theta_{i}\right)$

$$
\left.\underset{\theta_{-i}}{E}\left(\mathcal{U}_{i}\left(\sigma_{i}\left(m_{i}^{a}, m_{i}^{p *}\left(m_{i}^{a}, m_{-i}^{a *}\left(\theta_{-i}\right)\right)\right), \sigma_{-i}\left(m_{-i}^{a *}\left(\theta_{-i}\right), m_{-i}^{p *}\left(m_{i}^{a}, m_{-i}^{a *}\left(\theta_{-i}\right)\right)\right)\right), \theta_{i}\right) \mid \theta_{i}\right)
$$

(B1)

$$
\left.\geq \underset{\theta_{-i}}{E}\left(\mathcal{U}_{i}\left(\sigma_{i}\left(\hat{m}_{i}^{a}, m_{i}^{p *}\left(\hat{m}_{i}^{a}, m_{-i}^{a *}\left(\theta_{-i}\right)\right)\right), \sigma_{-i}\left(m_{-i}^{a *}\left(\theta_{-i}\right), m_{-i}^{p *}\left(\hat{m}_{i}^{a}, m_{-i}^{a *}\left(\theta_{-i}\right)\right)\right)\right), \theta_{i}\right) \mid \theta_{i}\right) \quad \forall \hat{m}_{i}^{a} \in \mathcal{M}_{i}^{a} ;
$$

- Agents accept their offers:

$$
\left.\underset{\theta_{-i}}{E}\left(\mathcal{U}_{i}\left(\sigma_{i}\left(m_{i}^{a}, m_{i}^{p *}\left(m_{i}^{a}, m_{-i}^{a *}\left(\theta_{-i}\right)\right)\right), \sigma_{-i}\left(m_{-i}^{a *}\left(\theta_{-i}\right), m_{-i}^{p *}\left(m_{i}^{a}, m_{-i}^{a *}\left(\theta_{-i}\right)\right)\right)\right), \theta_{i}\right) \mid \theta_{i}\right) \geq 0
$$

- The principal's reporting strategy $\mathbf{m}^{p *}\left(\mathbf{m}_{\mathbf{a}}\right)=\left(m_{1}^{p *}\left(\mathbf{m}_{\mathbf{a}}\right), . ., m_{n}^{p *}\left(\mathbf{m}_{\mathbf{a}}\right)\right)$ is any (pure) selection within his bestresponse correspondence:

(B3)

$$
\mathcal{V}\left(\sigma_{1}\left(m_{1}^{a}, m_{1}^{p *}\left(\mathbf{m}_{\mathbf{a}}\right)\right), \ldots, \sigma_{n}\left(m_{n}^{a}, m_{n}^{p *}\left(\mathbf{m}_{\mathbf{a}}\right)\right)\right) \geq \mathcal{V}\left(\sigma_{1}\left(m_{1}^{a}, \hat{m}_{1}^{p}\right), \ldots, \sigma_{n}\left(m_{n}^{a}, \hat{m}_{n}^{p}\right)\right) \quad \forall\left(\hat{m}_{1}^{p}, \ldots, \hat{m}_{n}^{p}\right) \in \mathcal{M}^{p}
$$


Proof of Lemma B.1. Take any arbitrary set of bilateral mechanisms $\mathcal{B}=\left(\mathcal{B}_{1}, \ldots, \mathcal{B}_{n}\right)$ with the corresponding array of distributions $\left(\sigma_{1}\left(m_{1}^{a}, m_{1}^{p}\right), \ldots, \sigma_{n}\left(m_{n}^{a}, m_{n}^{p}\right)\right)$. Consider also a perfect Bayesian continuation equilibrium following acceptance. Such continuation is a pair of strategy $\left\{\mathbf{m}^{a *}, \mathbf{m}^{p *}\right\}$ and a belief $\operatorname{system} d \mu\left(\theta \mid \mathbf{m}^{a}\right)$ that altogether satisfy the following conditions.

- The principal updates his beliefs on the agents' types following Bayes' rule whenever possible, i.e, when $\mathbf{m}^{a} \in \operatorname{supp} m_{1}^{a *}\left(\theta_{1}\right) \times \ldots \times \operatorname{supp} m_{n}^{a *}\left(\theta_{n}\right)$ for some $\left(\theta_{1}, \ldots, \theta_{n}\right)$. Otherwise, beliefs are arbitrary. Let $d \mu\left(\theta \mid \mathbf{m}^{a}\right)$ denote the updated belief system following any arbitrary message $m^{a}$.

- Given any such vector $m$ (either on- or off- equilibrium) and the corresponding posterior beliefs, the principal chooses messages $\mathbf{m}^{p *}\left(\mathbf{m}^{a}\right)$ in his best-response correspondence such that:

$$
\mathbf{m}^{p *}\left(\mathbf{m}^{a}\right) \in \arg \max _{\mathbf{m}^{p} \in \mathcal{M} p} \int_{\Theta} \mathcal{V}\left(\sigma_{1}\left(m_{1}^{a}, \hat{m}_{1}^{p}\right), \ldots, \sigma_{n}\left(m_{n}^{a}, \hat{m}_{n}^{p}\right)\right) d \mu\left(\theta \mid \mathbf{m}^{a}\right)
$$

Since in a private values context the agents' types do not enter directly into the principal's utility function, expectations do not matter and (B4) can be expressed as the pointwise optimization (B3).

- $A_{i}$ with type $\theta_{i}$ sends messages according to the mixed strategy $\mathbf{m}_{i}^{a *}\left(\theta_{i}\right)$ anticipating the principal's best response $\mathbf{m}^{p *}\left(\mathbf{m}^{a}\right)$. The mixed strategies $\left(m_{1}^{a *}\left(\theta_{1}\right), \ldots, m_{n}^{a *}\left(\theta_{n}\right)\right)$ form a Bayesian-Nash equilibrium which gives (B1). Acceptance then follows from (B2).

An array of bilateral contracts $\mathcal{B}=\left(\mathcal{B}_{1}, \ldots, \mathcal{B}_{n}\right)$ with the corresponding distributions of payments and outputs in each bilateral relationship $\left(\sigma_{1}\left(m_{1}^{a}, m_{1}^{p}\right), . ., \sigma_{n}\left(m_{n}^{a}, m_{n}^{p}\right)\right)$ and a continuation equilibrium $\left\{\mathbf{m}^{a *}, \mathbf{m}^{p *}\right\}$ altogether induce a (possibly stochastic) allocation:

$$
\left(\sigma_{1}\left(m_{1}^{a *}\left(\theta_{1}\right), m_{1}^{p *}\left(\mathbf{m}^{a *}(\theta)\right)\right), \ldots, \sigma_{n}\left(m_{n}^{a *}\left(\theta_{n}\right), m_{n}^{p *}\left(\mathbf{m}^{a *}(\theta)\right)\right)\right),
$$

where, in case of mixed reporting strategies, the notation $m_{i}^{p *}\left(\mathbf{m}^{a *}(\theta)\right)$ (respectively the notation $\sigma_{i}\left(m_{i}^{a *}\left(\theta_{i}\right), m_{i}^{p *}\left(\mathbf{m}^{a *}(\theta)\right)\right)$ denotes the distribution over $\mathcal{M}_{i}^{p}$ (resp. over $\mathcal{Q}_{i} \times \mathcal{T}_{i}$ ) induced by the strategies $\mathbf{m}^{p *}$ and $\mathbf{m}^{a *}$. Direct revelation mechanisms are helpful to characterize such allocations. A (bilateral) direct revelation mechanism $\tilde{\sigma}_{i}\left(\hat{\theta}_{i}, \hat{\theta}_{-i}\right)$ indeed specifies a distribution on $A_{i}$ 's payment and output as a function of reports $\left(\hat{\theta}_{i}, \hat{\theta}_{-i}\right)$ where the first item $\hat{\theta}_{i} \in \Theta$ is $A_{i}$ 's own report on his own type whereas $\hat{\theta}_{-i} \in \Theta^{n-1}$ is the principal's report on what he has learned from other agents. Let $\tilde{\sigma}=\left(\tilde{\sigma}_{1}, \ldots, \tilde{\sigma}_{n}\right)$ be a collection of such bilateral direct mechanisms. For further references, let also $\tilde{\sigma}_{k}\left(\hat{\theta}_{k},\left(\hat{\theta}_{i}, \hat{\theta}_{-i-k}\right)\right)$ denote the distribution of payments and outputs in the bilateral relationship between $A_{k}$ and the principal when the former reports $\hat{\theta}_{k}$, and the latter reports $\hat{\theta}_{i}$ on $A_{i}$ (for $i \neq k$ ) and $\hat{\theta}_{-i-k}$ on others. Formally, the distribution on transfers and outputs in the bilateral relationship between $A_{i}$ and the principal is given by:

$$
\tilde{\sigma}_{i}\left(\hat{\theta}_{i}, \hat{\theta}_{-i}\right)=\sigma_{i}\left(m_{i}^{a *}\left(\hat{\theta}_{i}\right), m_{i}^{p *}\left(\mathbf{m}^{a *}\left(\hat{\theta}_{i}, \hat{\theta}_{-i}\right)\right)\right)
$$

and we also have

$$
\tilde{\sigma}_{k}\left(\hat{\theta}_{k},\left(\hat{\theta}_{i}, \hat{\theta}_{-i-k}\right)\right)=\sigma_{k}\left(m_{k}^{a *}\left(\hat{\theta}_{k}\right), m_{k}^{p *}\left(\mathbf{m}^{a *}\left(\hat{\theta}_{i}, \hat{\theta}_{-i}\right)\right)\right) .
$$

PROPOSITION B.1: In a private values context, any allocation achieved at a continuation equilibrium $\left\{\mathbf{m}^{a *}, \mathbf{m}^{p *}\right\}$ with the offer and acceptance of the bilateral contracts $\mathcal{B}$ can also be implemented through a collection of bilateral direct mechanisms $\tilde{\sigma}=\left(\tilde{\sigma}_{1}, \ldots, \tilde{\sigma}_{n}\right)$ satisfying (B5) and (B6) and such that:

1) The agents' participation constraints hold:

$$
\left.\underset{\theta_{-i}}{E}\left(\mathcal{U}_{i}\left(\tilde{\sigma}_{1}\left(\theta_{1},\left(\theta_{i}, \theta_{-1-i}\right)\right)\right), . ., \tilde{\sigma}_{i}\left(\theta_{i}, \theta_{-i}\right), \ldots, \tilde{\sigma}_{n}\left(\theta_{n},\left(\theta_{i}, \theta_{-i-n}\right)\right), \theta_{i}\right) \mid \theta_{i}\right) \geq 0
$$

2) The agents' Bayesian incentive compatibility constraints hold:

$$
\left.\underset{\theta_{-i}}{E}\left(\mathcal{U}_{i}\left(\tilde{\sigma}_{1}\left(\theta_{1},\left(\theta_{i}, \theta_{-1-i}\right)\right)\right), . ., \tilde{\sigma}_{i}\left(\theta_{i}, \theta_{-i}\right), \ldots, \tilde{\sigma}_{n}\left(\theta_{n},\left(\theta_{i}, \theta_{-i-n}\right)\right), \theta_{i}\right) \mid \theta_{i}\right)
$$


(B8)

$$
\geq \underset{\theta_{-i}}{E}\left(\mathcal{U}_{i}\left(\tilde{\sigma}_{1}\left(\theta_{1},\left(\hat{\theta}_{i}, \theta_{-1-i}\right)\right), \ldots, \tilde{\sigma}_{i}\left(\hat{\theta}_{i}, \theta_{-i}\right), \ldots, \tilde{\sigma}_{n}\left(\theta_{n},\left(\hat{\theta}_{-i}, \hat{\theta}_{-i-n}\right)\right), \theta_{i}\right) \mid \theta_{i}\right) \quad \forall\left(\theta_{i}, \hat{\theta}_{i}, \theta_{-i}\right) ;
$$

3) The principal's ex post incentive compatibility constraints (EPIC) hold:

$$
\mathcal{V}\left(\tilde{\sigma}_{1}\left(\theta_{1}, \theta_{-1}\right), \ldots, \tilde{\sigma}_{i}\left(\theta_{i}, \theta_{-i}\right), \ldots, \tilde{\sigma}_{n}\left(\theta_{n}, \theta_{-n}\right)\right)
$$

$$
\geq \mathcal{V}\left(\tilde{\sigma}_{1}\left(\theta_{1}, \hat{\theta}_{-1}\right), \ldots, \tilde{\sigma}_{i}\left(\theta_{i}, \hat{\theta}_{-i}\right), \ldots, \tilde{\sigma}_{n}\left(\theta_{n}, \hat{\theta}_{-n}\right)\right) \quad \forall\left(\theta, \hat{\theta}_{-1}, \ldots, \hat{\theta}_{-n}\right)
$$

Proof of Proposition B.1. First, it is routine to check that the agents' Bayesian incentive constraints (B1) imply (B8). From the definition (B5) and the incentive constraint (B1), we get:

$$
\underset{\theta_{-i}}{E}\left(\mathcal{U}_{i}\left(\tilde{\sigma}_{1}\left(\theta_{1},\left(\theta_{i}, \theta_{-1-i}\right)\right), \ldots, \tilde{\sigma}_{i}\left(\theta_{i}, \theta_{-i}\right), \ldots, \tilde{\sigma}_{n}\left(\theta_{n},\left(\theta_{-i}, \theta_{-i-n}\right)\right), \theta_{i}\right) \mid \theta_{i}\right)=
$$

$\underset{\theta_{-i}}{E}\left(\mathcal{U}_{i}\left(\sigma_{1}\left(m_{1}^{a *}\left(\theta_{1}\right), m_{1}^{p *}\left(\mathbf{m}^{a *}\left(\theta_{i}, \theta_{-i}\right)\right)\right), ., \sigma_{i}\left(m_{i}^{a *}\left(\theta_{i}\right), m_{i}^{p *}\left(\mathbf{m}^{a *}\left(\theta_{i}, \theta_{-i}\right)\right)\right), ., \sigma_{n}\left(m_{n}^{a *}\left(\theta_{n}\right), m_{n}^{p *}\left(\mathbf{m}^{a *}\left(\theta_{i}, \theta_{-i}\right)\right)\right), \theta_{i}\right) \mid \theta_{i}\right)$

$\underset{\theta_{-i}}{E}\left(\mathcal{U}_{i}\left(\sigma_{1}\left(m_{1}^{a *}\left(\theta_{1}\right), m_{1}^{p *}\left(\mathbf{m}^{a *}\left(\hat{\theta}_{i}, \theta_{-i}\right)\right)\right), ., \sigma_{i}\left(m_{i}^{a *}\left(\hat{\theta}_{i}\right), m_{i}^{p *}\left(\mathbf{m}^{a *}\left(\hat{\theta}_{i}, \theta_{-i}\right)\right)\right), ., \sigma_{n}\left(m_{n}^{a *}\left(\theta_{n}\right), m_{n}^{p *}\left(\mathbf{m}^{a *}\left(\hat{\theta}_{i}, \theta_{-i}\right)\right)\right), \theta_{i}\right) \mid \theta_{i}\right)$

$(\mathrm{B} 10) \quad=\underset{\theta_{-i}}{E}\left(\mathcal{U}_{i}\left(\tilde{\sigma}_{1}\left(\theta_{1},\left(\hat{\theta}_{i}, \theta_{-1-i}\right)\right), \ldots, \tilde{\sigma}_{i}\left(\hat{\theta}_{i}, \theta_{-i}\right), \ldots, \tilde{\sigma}_{n}\left(\theta_{n},\left(\hat{\theta}_{i}, \theta_{-i-n}\right)\right), \theta_{i}\right) \mid \theta_{i}\right) \quad \forall\left(\theta_{i}, \hat{\theta}_{i}, \theta_{-i}\right)$

where the first and the last equalities follow from using (B5) and (B6) respectively and the middle inequality follows from (B1) using $\hat{m}_{i}^{a}=m_{i}^{a *}\left(\hat{\theta}_{i}\right)$.

Then, observe that the direct bilateral mechanisms are now acceptable when (B7) holds.

Turning now to the principal's ex post incentive constraints, observe now that using (B5) gives us: (B11)

$\mathcal{V}\left(\tilde{\sigma}_{1}\left(\theta_{1}, \theta_{-1}\right), \ldots, \tilde{\sigma}_{i}\left(\theta_{i}, \theta_{-i}\right), \ldots, \tilde{\sigma}_{n}\left(\theta_{n}, \theta_{-n}\right)\right)=\mathcal{V}\left(\sigma_{1}\left(m_{1}^{a *}\left(\theta_{1}\right), m_{1}^{p *}\left(\mathbf{m}^{a *}(\theta)\right)\right), \ldots, \sigma_{n}\left(m_{n}^{a}\left(\theta_{n}\right), m_{n}^{p *}\left(\mathbf{m}^{a *}(\theta)\right)\right)\right)$

$$
=\int \mathcal{V}\left(\sigma_{1}\left(m_{1}^{a}, m_{1}^{p *}\left(\mathbf{m}^{a}\right)\right), ., \sigma_{n}\left(m_{n}^{a}, m_{n}^{p *}\left(\mathbf{m}^{a}\right)\right)\right) d m_{1}^{a *}\left(m_{1}^{a} \mid \theta_{1}\right) \ldots d m_{n}^{a *}\left(m_{n}^{a} \mid \theta_{n}\right) .
$$

Using (B3), we know that for any $\mathbf{m}^{a}=\left(m_{1}^{a}, \ldots, m_{n}^{a}\right)$ :

$$
\mathcal{V}\left(\sigma_{1}\left(m_{1}^{a}, m_{1}^{p *}\left(\mathbf{m}^{a}\right)\right), \ldots, \sigma_{n}\left(m_{n}^{a}, m_{n}^{p *}\left(\mathbf{m}^{a}\right)\right)\right) \geq \mathcal{V}\left(\sigma_{1}\left(m_{1}^{a}, \hat{m}_{1}^{p}\right), \ldots, \sigma_{n}\left(m_{n}^{a}, \hat{m}_{n}^{p}\right)\right) \quad \forall \hat{\mathbf{m}}^{p}=\left(\hat{m}_{1}^{p}, \ldots, \hat{m}_{n}^{p}\right) .
$$

Taking in particular $\hat{m}_{i}^{p}=m_{i}^{p *}\left(m_{i}^{a}, \hat{\mathbf{m}}_{-i}^{a}\right)$ where $\hat{\mathbf{m}}_{-i}^{a} \in \operatorname{supp} m_{-i}^{a *}\left(\hat{\theta}_{-i}\right)$ for some $\hat{\theta}_{-i}$, we get:

$V\left(\sigma_{1}\left(m_{1}^{a}, m_{1}^{p *}\left(\mathbf{m}^{a}\right)\right), \ldots, \sigma_{n}\left(m_{n}^{a}, m_{n}^{p *}\left(\mathbf{m}^{a}\right)\right)\right) \geq \mathcal{V}\left(\sigma_{1}\left(m_{1}^{a}, m_{1}^{p *}\left(m_{1}^{a}, \hat{\mathbf{m}}_{-1}^{a}\right)\right), \ldots, \sigma_{n}\left(m_{n}^{a}, m_{n}^{p *}\left(m_{n}^{a}, \hat{\mathbf{m}}_{-n}^{a}\right)\right)\right)$.

By integrating over the relevant mixtures, we thus obtain:

$$
\int \mathcal{V}\left(\sigma_{1}\left(m_{1}^{a}, m_{1}^{p *}\left(\mathbf{m}^{a}\right)\right), . . \sigma_{n}\left(m_{n}^{a}, m_{n}^{p *}\left(\mathbf{m}^{a}\right)\right)\right) d m_{1}^{a *}\left(m_{1}^{a} \mid \theta_{1}\right) \ldots d m_{n}^{a *}\left(m_{n}^{a} \mid \theta_{n}\right) \geq
$$

(B12)

$\int \mathcal{V}\left(\sigma_{1}\left(m_{1}^{a}, m_{1}^{p *}\left(m_{1}^{a}, \hat{\mathbf{m}}_{-1}^{a}\right)\right), ., \sigma_{n}\left(m_{n}^{a}, m_{n}^{p *}\left(m_{n}^{a}, \hat{\mathbf{m}}_{-n}^{a}\right)\right)\right) d m_{1}^{a *}\left(m_{1}^{a} \mid \theta_{1}\right) d m_{-1}^{a *}\left(\hat{m}_{-1}^{a} \mid \hat{\theta}_{-1}\right) \ldots d m_{n}^{a *}\left(m_{n}^{a} \mid \theta_{n}\right) d m_{-n}^{a *}\left(\hat{m}_{-n}^{a} \mid \hat{\theta}_{-n}\right)$ 
Gathering (B11) and (B12), we finally get:

$$
\begin{aligned}
& \mathcal{V}\left(\tilde{\sigma}_{1}\left(\theta_{1}, \theta_{-1}\right), \ldots, \tilde{\sigma}_{i}\left(\theta_{i}, \theta_{-i}\right), \ldots, \tilde{\sigma}_{n}\left(\theta_{n}, \theta_{-n}\right)\right) \\
& \geq \mathcal{V}\left(\sigma_{1}\left(m_{1}^{a *}\left(\theta_{1}\right), m_{1}^{p *}\left(\mathbf{m}^{a *}\left(\theta_{1}, \hat{\theta}_{-1}\right)\right)\right), \ldots, \sigma_{n}\left(m_{n}^{a}\left(\theta_{n}\right), m_{n}^{p *}\left(\mathbf{m}^{a *}\left(\theta_{n}, \hat{\theta}_{-n}\right)\right)\right)\right) \\
& =\mathcal{V}\left(\tilde{\sigma}_{1}\left(\theta_{1}, \hat{\theta}_{-1}\right), \ldots, \tilde{\sigma}_{i}\left(\theta_{i}, \hat{\theta}_{-i}\right), \ldots, \tilde{\sigma}_{n}\left(\theta_{n}, \hat{\theta}_{-n}\right)\right) \quad \forall\left(\theta, \hat{\theta}_{-1}, \ldots, \hat{\theta}_{-n}\right)
\end{aligned}
$$

where the last equality follows from using (B5).

Proposition 1 in the text is a direct consequence of the more general statement Proposition B.1.

The Revelation Principle obtained in this dynamic environment with limited commitment differs from that presented in Myerson (1986) ${ }^{61}$ in two respects. Following his general methodology for dynamic games of incomplete information, there is no loss of generality in using direct revelation mechanisms where informed players send reports on their information at any stage to a central mediator and then obey his recommendations (which may possibly involve communication strategies towards their principal which are mixtures as in Strausz, 2006, for instance). Here, we keep decentralized communication and the final allocation is implemented with an array of direct revelation mechanisms. Second, with mediated communication and the corresponding centralized direct mechanisms, the agents use pure strategies in reporting to the mediator who then recommends them to mix their reports to the principal. In our framework, it could also be a priori interesting to let agents misrepresent their types with some probability. However, mixing is worthless with private values. The principal's payoff does not depend directly on the agents' types but only indirectly through payments and outputs. The principal's beliefs on the agents' types following their reports do not affect how he chooses outputs at the last stage of the game. This leads to a simple version of the Revelation Principle where agents play pure strategies and the principal chooses an ex post optimal output. ${ }^{62}$

\section{For Online Publication. Appendix C: Alternative Assumptions}

This section first proposes two extensions of the framework developed in Section III. In the first one, we use a specific information structure, slightly different from that in the main text, and show that, with strong correlation, the incentive problem is no longer regular and the principal's opportunism may have almost no cost for the vertical structure. In the second scenario, we instead depart from the main text by investigating optimal contracts in the case of a zero-one decision. It allows us to give a clear upper bound on types correlation that is consistent with regularity of the incentive problem. Finally, this section also comes back to the framework of Section IV.B and study the irrelevance of extended mechanisms.

\section{C1. Strong Correlation}

We now consider the polar case of a strong correlation and show that EPIC may have much less impact in such context. To make this point as tractable as possible, we depart from our previous information structure and now adopt the following expression of conditional distributions:

$$
\tilde{F}\left(\theta_{-i} \mid \theta_{i}\right)= \begin{cases}(1-h) F\left(\theta_{-i}\right) & \text { if } \theta_{-i}<\theta_{i} \\ h+(1-h) F\left(\theta_{-i}\right) & \text { if } \theta_{-i} \geq \theta_{i}\end{cases}
$$

${ }^{61}$ See Myerson, R., 1986, “Multistage Games with Communication”, Econometrica, (54): p323-358.

${ }^{62}$ Notice that in Proposition B.1 as well as in Proposition 1, the direct mechanisms may be stochastic. However, in our analysis of the main text, each time the Revelation Principle is used to derive optimal contracts, the incentive problem is such that non-local incentive compatibility is never a binding constraint for the optimal mechanism when the incentive problem is regular. In a similar setting with one agent and a finite type set, it is known (see Strausz, R., 2006, "Deterministic vs Stochastic Mechanisms in Principal-Agent Models", Journal of Economic Theory, (128): p308-314.) that optimal mechanisms are deterministic, so that there is no loss of generality in restricting attention to deterministic direct mechanisms or deterministic nonlinear wholesale prices. 
where $h \in[0,1)$ and $F(\cdot)$ is still the unconditional cumulative distribution. In other words, the distribution puts a Dirac mass on the diagonal $\theta_{1}=\theta_{2}$. We will be particularly interested in the limiting case of perfect correlation where $h$ approaches 1 .

Ex post incentive compatible mechanisms are still of the form given in (9). In particular, let us consider the ex post incentive compatible mechanism $\left\{t_{i}^{h}(\theta), q_{i}^{h}(\theta)\right\}_{\theta \in \Theta^{n}}$ such that:

$$
t_{i}^{h}(\theta)=\left\{\begin{array}{l}
C\left(q^{f b}\left(\theta_{i}\right)\right)+h W^{f b}\left(\theta_{i}\right) \\
h W^{f b}\left(\theta_{i}\right)
\end{array} \quad \text { and } q_{i}^{h}(\theta)= \begin{cases}q^{f b}\left(\theta_{i}\right) & \text { if } \theta_{-i}=\theta_{i} \\
0 & \text { if } \theta_{-i} \neq \theta_{i}\end{cases}\right.
$$

where $W^{f b}\left(\theta_{i}\right)=R\left(q^{f b}\left(\theta_{i}\right)\right)-C\left(q^{f b}\left(\theta_{i}\right)\right)-\theta_{i} q^{f b}\left(\theta_{i}\right)$ is the (non-negative) first-best surplus with type $\theta_{i}$.

That mechanism yields payoff $h W^{f b}\left(\theta_{i}\right)$ to the principal when $A_{i}{ }^{\prime}$ type is $\theta_{i}$. It rewards agents only if their reports agree which, given the information structure, arises with positive probability only if they both tell the truth. The mechanism is also structured to extract all surplus from the agents. From this remark, we immediately get:

PROPOSITION C.1: Assume that the information structure is as in (C1). The principal's expected payoff with the mechanism $\left\{\left(t_{i}^{h}(\theta), q_{i}^{h}(\theta)\right)\right\}_{\theta \in \Theta^{n}}$ converges toward its first-best value $\underset{\theta_{i}}{\operatorname{E}}\left(W^{f b}\left(\theta_{i}\right)\right)$ as $h$ converges to one.

Proof of Proposition C.1. The mechanism $\left\{t_{i}^{h}(\theta), q_{i}^{h}(\theta)\right\}_{\theta \in \Theta^{n}}$ is ex post incentive compatible for the principal and gives him payoff $h E\left(W^{f b}\left(\theta_{i}\right)\right)$ when dealing with $A_{i}$. This payoff converges towards the first-best expected payoff as $h$ converges to one. It remains to be checked whether this mechanism is Bayesian incentive compatible and individually rational. First, participation constraints hold since, by telling the truth, $A_{i}$ with type $\theta_{i}$ gets:

$$
U_{i}\left(\theta_{i}\right)=h W^{f b}\left(\theta_{i}\right)+(1-h) \times 0-h W^{f b}\left(\theta_{i}\right)=0 .
$$

Second, Bayesian incentive compatibility constraints hold since the non-negativity of the first-best surplus implies:

$$
U_{i}\left(\theta_{i}\right)=0 \geq h \times 0+(1-h) \times 0-h W^{f b}\left(\hat{\theta}_{i}\right) \quad \forall \hat{\theta}_{i} \neq \theta_{i} .
$$

\section{C2. $0-1$ Projects}

We now suppose that the principal wants to procure a unitary project from each of his retailers. The net return on the project is thus linear and can be written as $S q_{i}$ where $q_{i} \in[0,1]$ now stands for the (verifiable) probability of undertaking the project (and where $R-C=S$ ). In such an environment, EPIC requires using a payment schedule which is linear in that probability:

$$
T_{i}\left(q_{i}, \hat{\theta}_{i}\right)=S q_{i}-H_{i}\left(\hat{\theta}_{i}\right) .
$$

We shall now assume that

$$
\theta_{i}<S \text { and } \varphi\left(\theta_{i}, \bar{\theta}\right)<S<\varphi\left(\theta_{i}, \underline{\theta}\right)
$$

The first condition above ensures that it is always optimal to realize the project under complete information while the second condition requires that the other agent's type must be sufficiently "bad news" so as to be the case under asymmetric information.

PROPOSITION C.2: Assume that

$$
\tilde{f}\left(\bar{\theta} \mid \theta_{i}\right)-\left(S-\theta_{i}\right) \tilde{f}_{\theta_{i}}\left(\bar{\theta} \mid \theta_{i}\right) \geq 0 \quad \forall \theta_{i} \in \Theta .
$$


Then the incentive problem is regular and the optimal decision rule satisfies:

$$
q_{i}^{s b}\left(\theta_{i}, \theta_{-i}\right)= \begin{cases}1 & \text { if } S \geq \varphi\left(\theta_{i}, \theta_{-i}\right), \\ 0 & \text { otherwise }\end{cases}
$$

Proof of Proposition C.2. Proceeding as in the Proof of Proposition 2 and assuming that the principal's problem is regular, but taking into account the linearity yields the following maximand:

$$
(\mathcal{P}): \max _{\mathbf{q}(\cdot) \in[0,1]} \int_{\Theta^{2}} \tilde{f}(\theta)\left(\sum_{i=1}^{2}\left(\left(1+\frac{F\left(\theta_{i}\right)}{f\left(\theta_{i}\right)} \frac{\tilde{f}_{\theta_{i}}\left(\theta_{-i} \mid \theta_{i}\right)}{\tilde{f}\left(\theta_{-i} \mid \theta_{i}\right)}\right)\left(S-\theta_{i}\right)-\frac{F\left(\theta_{i}\right)}{f\left(\theta_{i}\right)}\right) q_{i}(\theta)\right) d \theta .
$$

Pointwise optimization gives the optimal decision rule defined by (C4). From the fact that $\varphi\left(\theta_{i}, \theta_{-i}\right)$ is increasing in $\theta_{i}$ and decreasing in $\theta_{-i}$ and because (C2) holds, there exists a non-decreasing function $\eta\left(\theta_{i}\right)$ such that $S=\varphi\left(\theta_{i}, \eta\left(\theta_{i}\right)\right)$. Condition (C4) can finally be rewritten as:

$$
q_{i}^{s b}\left(\theta_{i}, \theta_{-i}\right)= \begin{cases}1 & \text { if } \theta_{-i} \geq \eta\left(\theta_{i}\right) \\ 0 & \text { otherwise }\end{cases}
$$

With this specification of the decision rule, we can write:

$$
U_{i}^{s b}\left(\theta_{i}\right)=\max _{\hat{\theta}_{i} \in \Theta} E\left(\left(S-\theta_{-i}\right) q_{i}^{s b}\left(\hat{\theta}_{i}, \theta_{-i}\right) \mid \theta_{i}\right)-H_{i}\left(\hat{\theta}_{i}\right)=\max _{\hat{\theta}_{i} \in \Theta}\left(S-\theta_{i}\right)\left(1-\tilde{F}\left(\eta\left(\hat{\theta}_{i}\right) \mid \theta_{i}\right)\right)-H_{i}\left(\hat{\theta}_{i}\right) .
$$

Because $U_{i}^{s b}\left(\theta_{i}\right)$ so defined is absolutely continuous, it is almost everywhere differentiable and at any point of differentiability, satisfies:

$$
\dot{U}_{i}^{s b}\left(\theta_{i}\right)=-\left(1-\tilde{F}\left(\eta\left(\theta_{i}\right) \mid \theta_{i}\right)\right)-\left(S-\theta_{i}\right) \tilde{F}_{\theta_{i}}\left(\eta\left(\theta_{i}\right) \mid \theta_{i}\right) .
$$

Moreover, absolute continuity also implies:

$$
U_{i}^{s b}\left(\theta_{i}\right)-U_{i}^{s b}\left(\hat{\theta}_{i}\right)=\int_{\theta_{i}}^{\hat{\theta}_{i}}\left(1-\tilde{F}\left(\eta\left(\tilde{\theta}_{i}\right) \mid \tilde{\theta}_{i}\right)+\left(S-\tilde{\theta}_{i}\right) \tilde{F}_{\theta_{i}}\left(\eta\left(\tilde{\theta}_{i}\right) \mid \tilde{\theta}_{i}\right)\right) d \tilde{\theta}_{i}
$$

Incentive compatibility follows when:

$$
U_{i}^{s b}\left(\theta_{i}\right)-U_{i}^{s b}\left(\hat{\theta}_{i}\right) \geq\left(S-\theta_{i}\right)\left(1-\tilde{F}\left(\eta\left(\hat{\theta}_{i}\right) \mid \theta_{i}\right)\right)-\left(S-\hat{\theta}_{i}\right)\left(1-\tilde{F}\left(\eta\left(\hat{\theta}_{i}\right) \mid \hat{\theta}_{i}\right)\right)
$$

$$
=\int_{\theta_{i}}^{\hat{\theta}_{i}}\left(1-\tilde{F}\left(\eta\left(\hat{\theta}_{i}\right) \mid \tilde{\theta}_{i}\right)+\left(S-\tilde{\theta}_{i}\right) \tilde{F}_{\theta_{i}}\left(\eta\left(\hat{\theta}_{i}\right) \mid \tilde{\theta}_{i}\right)\right) d \tilde{\theta}_{i} .
$$

Gathering (C7) and (C8), incentive compatibility holds when:

$$
\int_{\theta_{i}}^{\hat{\theta}_{i}}\left(\int_{\eta\left(\hat{\theta}_{i}\right)}^{\eta\left(\tilde{\theta}_{i}\right)}\left(\left(S-\tilde{\theta}_{i}\right) \tilde{f}_{\theta_{i}}\left(x \mid \tilde{\theta}_{i}\right)-\tilde{f}\left(x \mid \tilde{\theta}_{i}\right)\right) d x\right) d \tilde{\theta}_{i} \geq 0 .
$$

Because $\eta(\cdot)$ is non-decreasing, a sufficient condition for (C9) to hold is

$$
\tilde{f}\left(\theta_{-i} \mid \theta_{i}\right)-\left(S-\theta_{i}\right) \tilde{f}_{\theta_{i}}\left(\theta_{-i} \mid \theta_{i}\right) \geq 0 \quad \forall\left(\theta_{i}, \theta_{-i}\right) \in \Theta^{2} .
$$


Integrating this latter condition over $\left[\eta\left(\theta_{i}\right), \bar{\theta}\right]$, we obtain:

$$
1-\tilde{F}\left(\eta\left(\theta_{i}\right) \mid \tilde{\theta}_{i}\right)+\left(S-\theta_{i}\right) \tilde{F}_{\theta_{i}}\left(\eta\left(\theta_{i}\right) \mid \theta_{i}\right) \geq 0 \quad \forall \theta_{i} \in \Theta .
$$

This shows that the right-hand side of (C6) is negative and the participation constraint (16) is binding at the optimal contract as requested in a regular problem.

Because MLRP holds, $\frac{\tilde{f}_{\theta_{i}}\left(\theta_{-i} \mid \theta_{i}\right)}{\tilde{f}\left(\theta_{-i} \mid \theta_{i}\right)} \leq \frac{\tilde{f}_{\theta_{i}}\left(\bar{\theta} \mid \theta_{i}\right)}{\tilde{f}\left(\bar{\theta} \mid \theta_{i}\right)}$ for all $\theta_{-i}$ and, moreover, $\frac{\tilde{f}_{\theta_{i}}\left(\bar{\theta} \mid \theta_{i}\right)}{\tilde{f}\left(\bar{\theta} \mid \theta_{i}\right)}$ is non-negative. From this, it follows that a sufficient condition for (C10) is that it holds at $\theta_{-i}=\bar{\theta}$ as requested by (C3).

\section{C3. Extended Mechanisms with Secret Contracts}

We now investigate whether the principal would like to deviate to a richer class of bilateral contracts to possibly communicate with retailer $A_{i}$ the endogenous information he has on the private offers he makes to $A_{-i}$. Denote thus by $\mathcal{M}_{i}$ any arbitrary compact message space available to the principal to communicate with $A_{i}$ and by $\left\{T_{i}\left(q_{i}, m_{i}, \hat{\theta}_{i}\right)\right\}_{\left\{\hat{\theta}_{i} \in \Theta, m_{i} \in \mathcal{M}_{i}\right\}}$ a menu of so extended nonlinear schemes. We assume that $T_{i}\left(q_{i}, m_{i}, \hat{\theta}_{i}\right)$ is upper semi-continuous in $m_{i}$ to ensure existence of an optimum at the last stage of the game. Finally, denote by $\mathbf{m}^{*}(\hat{\theta})=\left(m_{1}^{*}(\hat{\theta}), m_{2}^{*}(\hat{\theta})\right)$ a selection within the principal's correspondence of best responses to the messages $\hat{\theta}=\left(\hat{\theta}_{1}, \hat{\theta}_{2}\right)$ sent by the agents. Ex post optimality for the principal implies:

$$
\left(\mathbf{q}^{*}(\theta), \mathbf{m}^{*}(\theta)\right) \in \arg \max _{\mathbf{q} \in \mathcal{Q}, \mathbf{m} \in \prod_{i=1}^{2}} \sum_{\mathcal{M}_{i}}^{2} T_{i}\left(q_{i}, m_{i}, \theta_{i}\right)
$$

where the maximum above is achieved by compactness of $\mathcal{M}_{i}$ and upper semi-continuity in $p_{i}$. Let us define the new direct mechanism $\left(t_{i}^{s}(\theta), q_{i}^{s}(\theta)\right)=\left(T_{i}\left(\mathbf{q}_{i}^{*}(\theta), m_{i}^{*}(\theta), \theta_{i}\right), q_{i}^{*}(\theta)\right)$. Such a mechanism does not use "extended" reports from the principal and satisfies the agent's Bayesian incentive compatibility constraints. The optimality condition (C12) becomes:

$$
\theta \in \arg \max _{\hat{\theta} \in \Theta^{2}} \sum_{i=1}^{2} t_{i}^{s}\left(\theta_{i}, \hat{\theta}_{-i}\right)
$$

The new mechanism $\left(t_{i}^{s}(\theta), q_{i}^{s}(\theta)\right)$ is thus ex post incentive compatible. This shows that there is no point in enlarging the set of mechanisms available to the principal. 